\title{
COFORMALITY AND RATIONAL HOMOTOPY GROUPS OF SPACES OF LONG KNOTS
}

\author{
Greg Arone, Pascal Lambrechts, Victor Turchin, and Ismar Volić
}

AbSTRACT. We show that the Bousfield-Kan spectral sequence which computes the rational homotopy groups of the space of long knots in $\mathbb{R}^{d}$ for $d \geq 4$ collapses at the $E^{2}$ page. The main ingredients in the proof are Sinha's cosimplicial model for the space of long knots and a coformality result for the little balls operad.

\section{Introduction}

A long knot is a smooth embedding $f: \mathbb{R} \hookrightarrow \mathbb{R}^{d}$ that coincides with a fixed linear embedding outside of a compact subset of $\mathbb{R}$. The space of long knots, equipped with the weak $\mathcal{C}^{\infty}$-topology, will be denoted by $\operatorname{Emb}\left(\mathbb{R}, \mathbb{R}^{d}\right)$. One similarly defines the space of long immersions of $\mathbb{R}$ into $\mathbb{R}^{d}$, and we will denote this space by $\operatorname{Imm}\left(\mathbb{R}, \mathbb{R}^{d}\right)$. In this paper, we consider the homotopy fiber

$$
\overline{\operatorname{Emb}}\left(\mathbb{R}, \mathbb{R}^{d}\right):=\operatorname{hofiber}\left(\operatorname{Emb}\left(\mathbb{R}, \mathbb{R}^{d}\right) \hookrightarrow \operatorname{Imm}\left(\mathbb{R}, \mathbb{R}^{d}\right)\right) .
$$

This space was studied, for example, in [21] (where it was denoted by $E_{d}$ ). It is known (see [21, Proposition 5.17] for example) that

$$
\overline{\operatorname{Emb}}\left(\mathbb{R}, \mathbb{R}^{d}\right) \simeq \operatorname{Emb}\left(\mathbb{R}, \mathbb{R}^{d}\right) \times \Omega^{2} S^{d-1},
$$

so that any information about the homotopy type of $\overline{\operatorname{Emb}}\left(\mathbb{R}, \mathbb{R}^{d}\right)$ translates directly into information about the homotopy type of $\operatorname{Emb}\left(\mathbb{R}, \mathbb{R}^{d}\right)$.

Our starting point is the existence of a certain cosimplicial space,

$$
\mathcal{K}^{\bullet}=\left(\mathcal{K}^{0} \rightleftarrows \mathcal{K}^{1} \rightleftarrows \mathcal{K}^{2} \cdots\right),
$$

whose homotopy totalization $\operatorname{Tot}\left(\mathcal{K}^{\bullet}\right)$ is homotopy equivalent to $\overline{\operatorname{Emb}}\left(\mathbb{R}, \mathbb{R}^{d}\right)$ for $d \geq$ 4. For each $n \geq 0, \mathcal{K}^{n}$ has the homotopy type of the configuration space of ordered $n$ tuples of distinct points in $\mathbb{R}^{d}$. Coface maps are, roughly speaking, doubling maps, and codegeneracy maps are projection maps which forget certain points. The existence of this cosimplicial space was originally suggested by Goodwillie ([11, Example 5.1.4]) and was subsequently proved by Sinha ([21] and [20]; see Section 4 in this paper for more details).

Received by the editors January 12, 2007.

2000 Mathematics Subject Classification. Primary: 57Q45; Secondary: 55P62, 55P48.

Key words and phrases. knot spaces, embedding calculus, formality, operads, Bousfield-Kan spectral sequence.

The first author was supported in part by the National Science Foundation grant DMS 0605073. The second author is Chercheur Qualifié au F.N.R..S. He also thank IHES, where part of his work on this project was done, for its support and hospitality. The third author was supported in part by the grants NSH-1972.2003.01, RFBR 05-01-01012a. The fourth author was supported in part by the National Science Foundation grant DMS 0504390. 
It follows that for any abelian group $\mathbf{k}$, there is an associated homology BousfieldKan spectral sequence with coefficients in $\mathbf{k}$ ( $\mathbf{k H B K S S}$ for short) with $E^{1}$ page given by $E_{p, q}^{1}=\mathrm{H}_{q}\left(\mathcal{K}^{-p} ; \mathbf{k}\right)$ and a differential $d^{1}$ that can easily be made explicit [20]. This spectral sequence converges to $\mathrm{H}_{q+p}\left(\overline{\operatorname{Emb}}\left(\mathbb{R}, \mathbb{R}^{d}\right) ; \mathbf{k}\right)$ for $d \geq 4$ [21, Theorem 7.2].

The main result of [13] says that this spectral sequence collapses at the $E^{2}$ page when $\mathbf{k}=\mathbb{Q}$. The goal of the present paper is to prove an analogous result for homotopy groups. Thus we consider the rational homotopy Bousfield-Kan spectral sequence $(\mathbb{Q} \pi \mathrm{BKSS}$ for short) whose first page is given by

$$
E_{p, q}^{1}=\pi_{q}\left(\mathcal{K}^{-p}\right) \otimes \mathbb{Q} \text {. }
$$

This spectral sequence converges to $\pi_{q+p}\left(\overline{\operatorname{Emb}}\left(\mathbb{R}, \mathbb{R}^{d}\right)\right) \otimes \mathbb{Q}$, again when $d \geq 4[20$, Theorem 7.1]. Since rational homotopy groups of configuration spaces are well understood thanks to Cohen and Gitler [6], the $E^{1}$ page of $\mathbb{Q} \pi$ BKSS can be easily computed. The coface maps in $\mathcal{K}^{\bullet}$ are also well understood $[18,20]$ so it is not hard to write explicit formulas for the differential $d^{1}$. Some low degree calculations in the $E^{2}$ page were carried out in [18].

The main result of this paper is the following

Theorem 1.1. If $d \geq 4$, the rational homotopy Bousfield-Kan spectral sequence associated to $\mathcal{K}^{\bullet}$, which computes $\pi_{*}\left(\overline{\operatorname{Emb}}\left(\mathbb{R}, \mathbb{R}^{d}\right)\right) \otimes \mathbb{Q}$, collapses at the $E^{2}$ page.

The proof of this theorem appears at the end of the paper. The philosophy behind it mimics the one behind the proof of the collapse of the $\mathbb{Q H B K S S}$ in [13]. The main idea in that paper was that the cosimplicial space $\mathcal{K}^{\bullet}$ behaves like a formal cosimplicial space. In other words, taking the singular chains (with rational coefficients) on $\mathcal{K}^{\bullet}$ gives a cosimplicial chain complex which behaves as if it were quasi-isomorphic to its homology, even if it is not strictly formal. Morally speaking, this is a consequence of the fact that $\mathcal{K}^{\bullet}$ is closely related to the little $d$-disks operad, which is formal by a theorem of Kontsevich [12, Theorem 2] (see also [14]). It is easy to show that the $\mathbb{Q} H B K S S$ of a formal cosimplicial space collapses at the $E^{2}$ page (see Proposition 3.6). However, the authors of [13] were unable to prove formality of $\mathcal{K}^{\bullet}$. Instead, they built certain formal diagrams, equivalent in some sense to the truncations of $\mathcal{K}^{\bullet}$, and this was in the end enough to deduce the collapse of the homology spectral sequence.

For the proof of Theorem 1.1, we will now observe that the little $d$-disks operad looks like a coformal operad. Roughly speaking a space is formal when its rational homotopy type is determined by its rational cohomology algebra, and it is coformal when its rational homotopy type is determined by its rational homotopy Lie algebra (see [16]). The first technical difficulty we will encounter is in properly defining a coformal operad in the category of spaces (such as the little $d$-disks operad). The reason this is not straightforward is that coformality concerns simply-connected pointed spaces, while the little $d$-disks operad is unpointed. Because of this, we will instead work directly with the cosimplicial space $\mathcal{K}^{\bullet}$ but will again not be able to prove directly that it is coformal. Instead, we will build another cosimplicial space, $\left|\chi^{\bullet}\right|$, which is coformal by construction, and which is equivalent to $\mathcal{K}^{\bullet}$ as far as the Bousfield-Kan spectral sequences and totalization are concerned. We will make heavy use of the fact that both $\operatorname{Tot}\left(\mathcal{K}^{\bullet}\right)$ and $\operatorname{Tot}\left|\chi^{\bullet}\right|$ are H-spaces (this is so because both cosimplicial spaces come from multiplicative operads). This will be enough to prove Theorem 1.1, because the $\mathbb{Q} \pi \mathrm{BKSS}$ of a coformal cosimplicial space collapses at the 
$E^{2}$ page (Corollary 3.8). This line of argument is summarized in Theorem 4.1 which gives a somewhat specialized criterion for the collapse of a $\mathbb{Q} \pi$ BKSS .

A possible extension of our present results is suggested by the work in [1]. In that paper, it was proved that relative formality of the little disks operad implies the collapse of a certain spectral sequence computing the rational homology of the embedding space, $\overline{\operatorname{Emb}}\left(M, \mathbb{R}^{d}\right)$, for a general manifold $M$ (under some assumptions on the codimension of $M$ in $\mathbb{R}^{d}$ ). This considerably extends the collapse result for the rational homology of the space of knots, which is essentially the case when $M$ is 1-dimensional. We speculate that the coformality of the little disk operad can be used to prove that an analogous spectral sequence for the rational homotopy of $\overline{\operatorname{Emb}}\left(M, \mathbb{R}^{d}\right)$ collapses under suitable assumptions on codimension. We hope to come back to this in a future paper.

This paper is organized as follows: In Section 2 we review some classical results from stable and unstable rational homotopy theory and recall the notions of formal and coformal diagrams. In Section 3 we study the Bousfield-Kan spectral sequences from the rational homotopy viewpoint and prove the collapsing results for (co)formal cosimplicial spaces. In Section 4 we build a coformal cosimplicial space $\left|\chi^{\bullet}\right|$ whose rational homotopy theory is analogous to that of $\mathcal{K}^{\bullet}$ and deduce the collapsing result for the associated $\mathbb{Q} \pi \mathrm{BKSS}$.

\section{Stable and unstable rational homotopy theory}

In this section we review some well-known concepts and results from rational homotopy theory. The definitive reference for this subject (at least for the unstable version) is [9]. We also prove some folklore results for which we could not find a reference.

Fix a field $\mathbf{k}$ of characteristic zero. We will need the following categories and functors:

- DGL (and DGL D $_{1}$, the category of (1-reduced) graded Lie algebras over $\mathbf{k}$ as in $[17$, p. 209] or $[9]$;

- The forgetful functor $L \mapsto \mathrm{f} L$ from DGL to chain complexes;

- $\mathrm{CDGC}_{2}$, the category of 2-reduced augmented cocommutative differential graded coalgebras as in [17, p. 209] or [9];

- CDGA, the category of commutative differential graded algebras;

- sSet, the category of simplicial sets;

- Top, the category of topological spaces;

- The Quillen and Cartan-Chevalley-Eilenberg functors

$$
\mathcal{L}: \mathrm{CDGC}_{2} \rightleftarrows \mathrm{DGL}_{1}: \mathcal{C}
$$

as defined in $[17$, appendix B] or $[9, \S 22]$. These functors are homotopy inverses of each other;

- For a 2-reduced CDGC, $C$, the dual $\# C:=\operatorname{Hom}(C, \mathbf{k})$ is a CDGA so one gets a contravariant functor

$$
\text { \#: } \mathrm{CDGC}_{2} \longrightarrow \mathrm{CDGA}
$$

- The functor of piecewise polynomial forms,

$$
A_{P L}=A_{P L}(-; \mathbf{k}): \text { sSets } \longrightarrow \text { CDGA, }
$$


defined in $[9, \S 10(\mathrm{c})]$.

- The Sullivan realization contravariant functor,

$$
\langle-\rangle: \text { CDGA } \longrightarrow \text { sSets, }
$$

defined in $[9, \S 17(\mathrm{c})]$ as an adjoint of $A_{P L}$. We define the realization of a 1-reduced DGL $L$ as

$$
\langle L\rangle:=\langle \# \mathcal{C}(L)\rangle
$$

- The spatial realizations

$$
|-|: \text { CDGA } \rightarrow \text { Top, and } \quad|-|: \mathrm{DGL}_{1} \rightarrow \text { Top }
$$

defined as the composition of $\langle-\rangle$ with the geometric realization of simplical sets $[9, \S 17$ (d)]. Notice that for a 1-reduced DGL $L$, the space $|L|$ is pointed by the realization of the zero-map $0 \rightarrow L$.

For a 1-reduced DGL, $L$, we have an isomorphism of graded Lie algebras $\mathrm{H}_{*}(L) \cong$ $\pi_{*}(\Omega|L|)$, so there is a shift of degree $\mathrm{H}_{*}(L) \cong \pi_{*+1}(|L|)$.

When two differential objects, $A$ and $B$, are linked by a zig-zag of quasi-isomorphisms we will write $A \simeq B$ and we will say that they are weakly equivalent. If $A$ is a CDGA, it is not true in general that $A_{P L}(|A|) \simeq A$, but it is the case when $A$ is a Sullivan algebra, [9, Theorem 17.10 (ii) ], or more generally for a cofibrant CDGA, $[3, \S 8]$. We also have the following

Lemma 2.1. Let $L$ be a 1-reduced DGL. Then

- $A_{P L}(|L|)$ is naturally weakly equivalent to $\# \mathcal{C}(L)$;

- $|-|: \mathrm{DGL}_{1} \rightarrow$ Top preserves weak equivalences.

Proof. By definition, $A_{P L}(|L|)=A_{P L}(|\# \mathcal{C}(L)|)$ and by construction, $\# \mathcal{C}(L)$ is a simply-connected Sullivan algebra. Theorem 17.10 (ii) of [9] gives the weak equivalence from the first statement and naturality follows from the discussion at the beginning of Section 17 (d) in [9]. The second part is a consequence of the fact that \# and $\mathcal{C}$ preserve weak equivalences as well as $|-|$ for morphisms between homologically simply-connected Sullivan algebras.

A space $X$ is called formal if its rational homotopy type is determined by its rational cohomology, more precisely if $A_{P L}(X)$ is weakly equivalent to $\mathrm{H}^{*}(X ; \mathbb{Q})$ [8]. Dually, a space $X$ is called coformal if its rational homotopy type is determined by its rational homotopy Lie algebra, $\pi_{*}(\Omega X) \otimes \mathbb{Q}[16]$. This algebra, by CartanSerre Theorem, is isomorphic to the primitive part of the rational homology of its loop space, $\mathrm{PH}_{*}(\Omega X ; \mathbb{Q})$. This can be generalized to diagrams and to other fields of characteristic 0 as follows.

Definition 2.2. Let $I$ be a small category and let $\mathbf{k}$ be a field of characteristic 0 .

- A diagram $X: I \rightarrow$ Top of spaces is said to be formal over $\mathbf{k}$ if $A_{P L}(X ; \mathbf{k})$ is linked to $\mathrm{H}^{*}(X ; \mathbf{k})$ by a chain of natural quasi-isomorphisms.

- A diagram $X: I \rightarrow$ Top of pointed simply-connected spaces is said to be coformal if $A_{P L}(X ; \mathbf{k})$ is linked to $\# \mathcal{C}\left(\mathrm{P} \mathrm{H}_{*}(\Omega X ; \mathbf{k})\right)$ by a chain of natural quasi-isomorphisms, where $\mathrm{PH}_{*}(-; \mathbf{k})$ stands for the primitive part of the homology. 
We now review some stable rational homotopy theory. Our goal is to prove that every rational simply-connected loop space is naturally equivalent to an infinite loop space (see Corollary 2.6). Let Spectra $\mathbb{Q}_{\mathbb{Q}}$ be the category of rational spectra (not necessarily connective and which are represented by a sequence of pointed simplicial sets), and let $\mathrm{Ch}_{\mathbb{Q}}$ be the category of unbounded chain complexes of $\mathbb{Q}$-modules. It is well known that there is a Quillen equivalence

$$
\|-\|: \mathrm{Ch}_{\mathbb{Q}} \rightleftarrows \text { Spectra }_{\mathbb{Q}}: \Lambda .
$$

One reference for this equivalence is [19], especially Appendix B (after the proof of Corollary B.1.8), specialized to $\underline{A}=\mathbb{Q}$ in the notation of that paper. Note that our notion of rational spectra corresponds to what Schwede and Shipley call in [19] naive $\mathrm{H} \mathbb{Q}$-spectra (as opposed to symmetric spectra). The spectral realization functor $\|-\|$ is denoted in [19, p.147] by $\mathcal{H}$ and it is defined as follows. Let $W: \mathrm{Ch}_{\mathbb{Q}} \rightarrow$ sSet be the truncation of a chain complex to a non-negatively graded one, followed by the Dold-Kan functor from non-negatively graded chain complexes to simplicial $\mathbb{Q}$-vector spaces. Then for a chain complex $C$, define

$$
\|C\|:=\left\{W\left(s^{n} C\right)\right\}_{n=0,1,2 \ldots}
$$

where $s^{n} C$ is the $n$-fold suspension of $C$. It is well-known that the sequence of simplicial sets $\left\{W\left(s^{n} C\right)\right\}$ forms an $\Omega$-spectrum.

Lemma 2.3. Let $L$ be a 1-reduced DGL. If $L$ is abelian (i.e. the bracket is zero) then $\langle L\rangle$ is naturally weakly equivalent to $W(s L)$ where $s L$ is the suspension of the underlying chain complex of $L$.

Proof. By definition, $\langle L\rangle=\langle \# \mathcal{C}(L)\rangle$ and $\# \mathcal{C}(L)=(\wedge Z, d)$ where $Z$ is the suspension of the dual of $L$. Since the bracket is zero, the differential is induced only by the dual of the differential on $L$, and it is hence determined by a linear map $d: Z \rightarrow Z$. The Sullivan realization of $(\wedge Z, d)$ is defined in $[9, \S 17$ (c)] by

$$
\langle(\wedge Z, d)\rangle=\operatorname{Hom}_{\mathrm{CDGA}}\left((\wedge Z, d), A_{P L} \bullet\right),
$$

where $A_{P L} \bullet$ is the simplicial CDGA defined in $[9, \S 10$ (c)].

Let $N \Delta[n]$ be the normalized chain complex of the standard simplicial set $\Delta[n]$, as in [19, p.147], and let $C_{P L} \bullet$ be the rational dual of $N \Delta[\bullet]$,

$$
C_{P L} \cong \operatorname{Hom}_{\mathbb{Z}-\operatorname{Mod}}(N \Delta[\bullet], \mathbb{Q}),
$$

defined in $[9, \S 10(\mathrm{~d})]$. By $[9$, Theorem 10.15] there is a weak equivalence of simplicial cochain complexes, $\oint: A_{P L} \stackrel{\cong}{\rightarrow} C_{P L}$.

Using the fact that $d$ is linear, we have the following natural weak equivalence

$$
\begin{aligned}
\langle(\wedge Z, d)\rangle & =\operatorname{Hom}_{\mathrm{CDGA}}\left((\wedge Z, d), A_{P L}\right) \cong \operatorname{Hom}_{\mathrm{Ch}}\left((Z, d), A_{P L \bullet}\right) \stackrel{\oint_{*}}{\simeq} \\
& \simeq \operatorname{Hom}_{\mathrm{Ch}_{\mathbb{Q}}}\left((Z, d), C_{P L \bullet} \cong \operatorname{Hom}_{\mathrm{Ch}_{\mathbb{Q}}}\left((Z, d), \operatorname{Hom}_{\mathbb{Z}-\mathrm{Mod}}(N \Delta[\bullet], \mathbb{Q})\right) \cong\right. \\
& \cong \operatorname{Hom}_{\mathrm{Ch}_{\mathbb{Q}}}\left((Z, d), \operatorname{Hom}_{\mathrm{Ch}}(N \Delta[\bullet \bullet \mathbb{Q}, \mathbb{Q})) \cong\right. \\
& \cong \operatorname{Hom}_{\mathrm{Ch}_{\mathbb{Q}}}\left(N \Delta[\bullet], \operatorname{Hom}_{\mathrm{Ch}_{\mathbb{Q}}}((Z, d), \mathbb{Q})\right) \cong \\
& \cong \operatorname{Hom}_{\mathrm{Ch}_{\mathbb{Q}}}(N \Delta[\bullet] \otimes \mathbb{Q}, s L) \cong \operatorname{Hom}_{\mathrm{Ch}_{\mathbb{Z}}}(N \Delta[\bullet], s L)=W(s L) .
\end{aligned}
$$

Recall the functor $\Omega^{\infty}:$ Spectra $_{\mathbb{Q}} \rightarrow$ Top $_{*}$ from rational spectra to pointed spaces. 
Corollary 2.4. If $L$ is an abelian 1-reduced $D G L$, there is a natural equivalence $|L| \simeq \Omega^{\infty}\|s L\|$.

Proof. Since $\|s L\|=\left(W\left(s^{n+1} L\right)\right)_{n \geq 0}$ is an $\Omega$-spectrum, it follows that $\Omega^{\infty}\|s L\|$ is the geometric realization of the zero-th simplicial set $W(s L)$ which, by Lemma 2.3, is naturally equivalent to $|L|$.

Proposition 2.5. Let $L$ be a 2-reduced $D G L$ and let $s^{-1} L$ be its desuspension with zero bracket, which is an abelian 1-reduced DGL. Then there is a natural weak equivalence $\Omega|L| \simeq\left|s^{-1} L\right|$.

Proof. Let $A(L)$ be an acyclic 1-reduced DGL defined as follows. As a graded vector space, $A(L)=L \oplus s^{-1} L$ and the bracket in $L$ is extended to $A(L)$ by $\left[s^{-1} x, y\right]=$ $\frac{1}{2} s^{-1}[x, y]$ and $\left[s^{-1} x, s^{-1} y\right]=0$ for $x, y \in L$.

Denote by $\partial$ the differential in $L$. The differential $D$ in $A(L)$ is then defined by $D(x)=\partial(x)-s^{-1} x$ and $D\left(s^{-1} x\right)=s^{-1} \partial(x)$ for $x \in L$. It is easy to see that $A(L)$ is a well-defined acyclic DGL and we have a short exact sequence

$$
s^{-1} L \hookrightarrow A(L) \rightarrow L,
$$

natural in $L$. By [9, Proposition 17.9], the spatial realization of this DGL sequence gives a fibration $|A(L)| \rightarrow|L|$ with fiber $\left|s^{-1} L\right|$. Since $|A(L)|$ is contractible, the associated connecting map (more precisely, a zig-zag of natural weak equivalences) $\partial: \Omega|L| \stackrel{\simeq}{\rightrightarrows}\left|s^{-1} L\right|$ is the desired natural weak equivalence.

Recall that $\mathrm{f} L$ is the underlying chain complex of $L$. The following is an immediate consequence of Corollary 2.4 and Proposition 2.5.

Corollary 2.6. If $L$ is a 2-reduced $D G L$, then $\Omega|L|$ and $\Omega^{\infty}\|\mathrm{f} L\|$ are naturally weakly equivalent.

\section{Spectral sequences associated to cosimplicial objects}

Unless stated otherwise, in this section "chain complex" means "non-negative chain complex".

For a cosimplicial Abelian group $A^{\bullet}$, let $\mathrm{N}^{\bullet} A$ be the associated normalized cochain complex. Explicitly,

$$
\mathrm{N}^{p} A:=A^{p} \cap \operatorname{ker} s^{0} \cap \ldots \cap \operatorname{ker} s^{p-1}
$$

where $s^{0} \ldots, s^{p-1}$ are the codegeneracy homomorphisms from $A^{p}$ to $A^{p-1}$. The coboundary homomorphism $d^{p}: \mathrm{N}^{p} A \rightarrow \mathrm{N}^{p+1} A$ is induced from the alternating sum of the coface maps in $A^{\bullet}$.

Now let $\mathcal{V}=V_{*}^{\bullet}$ be a cosimplicial chain complex. We define a tower of partial totalizations, $\left\{\operatorname{Tot}^{n}(\mathcal{V})\right\}_{n=0}^{\infty}$ as follows. $\operatorname{Tot}^{n}(\mathcal{V})$ is the total complex of the bicomplex obtained by applying $\mathrm{N}^{\bullet}$ to $\mathcal{V}$ degree-wise, and truncating in cosimplicial dimension $n$. More explicitly,

$$
\operatorname{Tot}^{n}(\mathcal{V})_{j}=\prod_{p=0}^{n}\left(\mathrm{~N}^{p} V\right)_{p+j},
$$

and $\operatorname{Tot}^{n}(\mathcal{V})$ is equipped with the total differential obtained in the usual way from the "vertical" differential $\mathrm{N}^{p} \partial_{q}: \mathrm{N}^{p} V_{q} \rightarrow \mathrm{N}^{p} V_{q-1}$ and the "horizontal" differential $d_{q}^{p}: \mathrm{N}^{p} V_{q} \rightarrow \mathrm{N}^{p+1} V_{q}$. The motivation for this definition of totalization of cosimplicial 
chain complexes is that it corresponds, under the Dold-Kan functor from chain complexes to simplicial Abelian groups, to the usual totalization of cosimplicial simplicial spaces. This is essentially Lemma 2.2 of [2].

Remark. It is well-known that for a group-like cosimplicial object, totalization is homotopy equivalent to "homotopy totalization", which is defined to be the homotopy limit of the cosimplicial diagram [4]. In particular, totalization of a cosimplicial simplicial Abelian group always has the "right" homotopy type, and the same is true for totalization of cosimplicial chain complexes.

There are natural surjective homomorphisms $\operatorname{Tot}^{n}(\mathcal{V}) \longrightarrow \operatorname{Tot}^{n-1}(\mathcal{V})$. The inverse limit of the resulting tower is denoted $\operatorname{Tot}(\mathcal{V})$. Explicitly,

$$
\operatorname{Tot}(\mathcal{V})_{j}=\prod_{p=0}^{\infty} \mathrm{N}^{p} V_{p+j} .
$$

The filtration of Tot by $\operatorname{Tot}^{n}$ gives rise to a spectral sequence abutting to $\mathrm{H}_{*}(\operatorname{Tot}(\mathcal{V}))$. We call it the Bousfield-Kan spectral sequence associated to the cosimplicial chain complex $\mathcal{V}$. Many of the standard spectral sequences in topology that are called "Bousfield-Kan spectral sequence" are obtained by first applying some functor into chain complexes to a cosimplicial object, to get a cosimplicial chain complex, and then applying the above construction. We will recall the particulars of two such spectral sequences below.

Definition 3.1. A cosimplicial chain complex $\mathcal{V}$ is called formal if it is weakly equivalent as a cosimplicial chain complex to its homology, $\mathrm{H}(\mathcal{V})$.

The main point we want to make in this section is contained in the following proposition.

Proposition 3.2. Let $\mathcal{V}$ be a formal cosimplicial chain complex. Then the associated Bousfield-Kan spectral sequence collapses at $E^{2}$.

The proof of the proposition follows immediately from the following three obvious lemmas.

Lemma 3.3. Suppose that a cosimplicial chain complex $\mathcal{V}$ splits as a product, i.e., there is a zig-zag of objectwise weak equivalences connecting the two cosimplicial chain complexes

$$
\mathcal{V} \simeq \prod_{i=0}^{\infty} \mathcal{W}_{i}
$$

Then the Bousfield-Kan spectral sequence of $\mathcal{V}$ is isomorphic to the product of the spectral sequences associated to $\mathcal{W}_{i}$. In particular, if each one of the Bousfield-Kan spectral sequences for $\mathcal{W}_{i}$ collapses at $E^{2}$, then so does the spectral sequence for $\mathcal{V}$.

Lemma 3.4. If a cosimplicial chain complex $\mathcal{V}$ is formal, then there is a zig-zag of weak equivalences

$$
\mathcal{V} \simeq \prod_{i=0}^{\infty} \mathrm{H}_{i}(\mathcal{V})
$$

where $\mathrm{H}_{i}\left(V^{p}\right)$ is defined to be a chain complex having the $i$-th homology of $V^{p}$ in dimension $i$, and zero in all other dimensions. 
Lemma 3.5. If $\mathcal{W}$ is a cosimplicial chain complex where all the chain complexes are concentrated in a fixed dimension $i$, then the associated Bousfield-Kan spectral sequence collapses at $E^{2}$ for dimensional reasons.

The rest of this section is devoted to the study of two particular manifestations of Proposition 3.2 in rational homotopy theory. The first instance is the homology Bousfield-Kan spectral sequence of a formal cosimplicial space. Let $X^{\bullet}$ be a cosimplicial space. Applying to $X^{\bullet}$ the singular chains functor $\mathrm{C}_{*}$, or the dual of the functor $A_{P L}$, we obtain a cosimplicial chain complex. As explained in [2, Section 2], the homology Bousfield-Kan spectral sequence associated to $X^{\bullet}$ is the spectral sequence associated to cosimplicial chain complex $C_{*}\left(X^{\bullet}\right)$, or $\# A_{P L}\left(X^{\bullet}\right)$. This immediately implies the following

Proposition 3.6. If $X^{\bullet}$ is a $\mathbf{k}$-formal cosimplicial space then the associated $\mathbf{k} H B K S S$ collapses at the $E^{2}$ page.

Proof. As $X^{\bullet}$ is formal, so is $C_{*}\left(X^{\bullet}\right)$ and Proposition 3.2 applies.

The second instance, and the more important one in this paper, is the homotopy Bousfield-Kan spectral sequence of a coformal cosimplicial space. First, we have a proposition saying that the homotopy groups of a cosimplicial DGL often can be calculated using only the underlying cosimplicial chain complex.

Proposition 3.7. Let $L^{\bullet}$ be a cosimplicial 2-reduced DGL. Consider the associated cosimplicial pointed space $\left|L^{\bullet}\right|$, and the underlying cosimplicial chain complex $\mathrm{f} L^{\bullet}$. There is an isomorphism, for $i \geq 0$,

$$
\pi_{i}\left(\Omega \operatorname{Tot}\left(\left|L^{\bullet}\right|\right)\right) \cong \mathrm{H}_{i}\left(\operatorname{Tot}\left(\mathrm{f} L^{\bullet}\right)\right)
$$

Proof. Keep in mind that $\Omega$ and $\Omega^{\infty}$ commute with homotopy limits. The same is true for the spectral realization $\|-\|$, because it is part of a Quillen equivalence. Using this, as well as the natural equivalence $\Omega \mid L^{\bullet} \stackrel{\cong}{\rightarrow} \Omega^{\infty}\left\|\mathrm{f} L^{\bullet}\right\|$ from Corollary 2.6, we obtain the following sequence of weak equvalences

$$
\begin{aligned}
\Omega \operatorname{Tot}\left|L^{\bullet}\right| & \simeq \Omega \operatorname{holim}_{\Delta}\left|L^{\bullet}\right| \simeq \operatorname{holim}_{\Delta} \Omega\left|L^{\bullet}\right| \simeq \\
& \simeq \operatorname{holim}_{\Delta} \Omega^{\infty}\left\|\mathrm{f} L^{\bullet}\right\| \simeq \Omega^{\infty} \operatorname{holim}_{\Delta}\left\|\mathrm{f} L^{\bullet}\right\| \simeq \\
& \simeq \Omega^{\infty}\left\|\operatorname{holim}_{\Delta} \mathrm{f} L^{\bullet}\right\| \simeq \Omega^{\infty}\left\|\operatorname{Tot}\left(\mathrm{f} L^{\bullet}\right)\right\| .
\end{aligned}
$$

Finally, using [19, Equation (B 1.9)] for the last isomorphism, we have the following chain of isomorphisms (where the second one only holds for $n \geq 0$ )

$$
\pi_{n}\left(\Omega \operatorname{Tot}\left(\left|L^{\bullet}\right|\right)\right) \cong \pi_{n}\left(\Omega^{\infty}\left\|\operatorname{Tot}\left(\mathrm{f} L^{\bullet}\right)\right\|\right) \cong \pi_{n}\left(\left\|\operatorname{Tot}\left(\mathrm{f} L^{\bullet}\right)\right\|\right) \cong H_{n}\left(\operatorname{Tot}\left(\mathrm{f} L^{\bullet}\right)\right) .
$$

Corollary 3.8. Let $L^{\bullet}$ be a cosimplicial 2-reduced DGL such that the underlying cosimplicial chain complex $\mathrm{f} L^{\bullet}$ is formal. If the $\pi B K S S$ for $\left|L^{\bullet}\right|$ converges to a graded vector space of finite type, then it collapses at the $E^{2}$ page in positive topological degrees.

Proof. It is enough to show that $\pi$ BKSS for $\Omega\left|L^{\bullet}\right|$ collapses in non-negative degrees. By Corollary 2.6, there is an equivalence of cosimplicial spaces

$$
\Omega\left|L^{\bullet}\right| \simeq \Omega^{\infty}\left\|\mathrm{f} L^{\bullet}\right\|
$$


so it is enough to prove that the homotopy Bousfield-Kan spectral sequence for $\Omega^{\infty}\left\|\mathrm{f} L^{\bullet}\right\|$ collapses at $E^{2}$. Let us now compare this spectral sequence with the homotopy spectral sequence for the cosimplicial spectrum $\|\mathrm{f} L \bullet\|$. Clearly the two spectral sequences have isomorphic $E^{1}$ pages and first differentials, so they have isomorphic $E^{2}$ pages. By assumption, the first spectral sequence converges to $\pi_{*}\left(\Omega\left|\operatorname{Tot}\left(L^{\bullet}\right)\right|\right)$. The second spectral sequence converges to $\mathrm{H}_{*}\left(\operatorname{Tot}\left(\mathrm{f} L^{\bullet}\right)\right)$. By Proposition 3.7, the two abutments are isomorphic in non-negative degrees. By assumption on the formality of $\mathrm{f} L^{\bullet}$ and Proposition 3.2, the second spectral sequence collapses. It follows that the first spectral sequence collapses in non-negative degrees.

Remark. Suppose $L^{\bullet}$ is a coformal cosimplicial 2-reduced DGL. It seems likely that the $\mathbb{Q} \pi$ BKSS for $\Omega\left|L^{\bullet}\right|$ agrees from the $E^{1}$ page with the homology spectral sequence of the double complex $\operatorname{Tot}\left(L^{\bullet}\right)$, but we have not been able to show this.

\section{Proof of Theorem 1.1}

As explained in the introduction, the moral of the proof of our main theorem is that $\mathcal{K}^{\bullet}$ "behaves like" a formal cosimplicial space and, for certain algebraic reasons related to its cohomology algebra and its homotopy Lie algebra, it also "behaves like" a coformal space, so its $\mathbb{Q} \pi$ BKSS collapses at the $E^{2}$ page. Because we could not prove that $\mathcal{K}^{\bullet}$ is indeed formal, we use a somewhat roundabout argument which could be summarized as follows: We consider the coformal cosimplicial space $\left|\chi^{\bullet}\right|$ associated to $\mathcal{K}^{\bullet}$. We show that for algebraic reasons it is also a formal cosimplicial space associated to $\mathcal{K}^{\bullet}$. So the $\mathbb{Q H B K S S}$ and $\mathbb{Q} \pi$ BKSS of this biformal cosimplicial space collapse, and we know by [13] that the $\mathbb{Q H B K S S}$ of $\mathcal{K}^{\bullet}$ collapses at the $E^{2}$ page. We deduce that $\mathrm{H}^{*}\left(\operatorname{Tot}\left(\mathcal{K}^{\bullet}\right) ; \mathbb{Q}\right) \cong \mathrm{H}^{*}\left(\operatorname{Tot}\left(\left|\chi^{\bullet}\right|\right) ; \mathbb{Q}\right)$. We also know that the totalizations are $\mathrm{H}$-spaces because the cosimplicial spaces are induced by multiplicative operads. We conclude that these totalizations in fact have the same rational homotopy type, and in particular the same rational homotopy groups. By coformality the $\mathbb{Q} \pi \mathrm{BKSS}$ of $\left|\chi^{\bullet}\right|$ collapses, and we deduce the same for $\mathcal{K}^{\bullet}$.

More precisely, the key argument is the following statement which will later be applied to $\mathcal{K}^{\bullet}$.

Theorem 4.1. Let $X^{\bullet}$ be a cosimplicial simply-connected space with rational homotopy groups of finite type and define the $D G L \chi^{\bullet}:=\mathrm{P} \mathrm{H}_{*}\left(\Omega X^{\bullet} ; \mathbb{Q}\right)$ with zero differentials. Suppose that

(1) The cosimplicial space $\left|\chi^{\bullet}\right|$ is formal and $\mathrm{H}^{*}\left(\left|\chi^{\bullet}\right| ; \mathbb{Q}\right) \cong \mathrm{H}^{*}\left(X^{\bullet} ; \mathbb{Q}\right)$;

(2) The $\mathbb{Q} H B K S S$ associated to $X^{\bullet}$ collapses at the $E^{2}$ page;

(3) The $\mathbb{Q} H B K S S$ and $\mathbb{Q} \pi B K S S$ associated to $X^{\bullet}$ and $\left|\chi^{\bullet}\right|$ converge to the rational homology and homotopy of their totalizations which are of finite type;

(4) $\operatorname{Tot}\left(X^{\bullet}\right)$ and $\operatorname{Tot}\left(\left|\chi^{\bullet}\right|\right)$ are $H$-spaces.

Then the $\mathbb{Q} \pi B K S S$ associated to $X^{\bullet}$ collapses at the $E^{2}$ page.

Proof. As $\left|\chi^{\bullet}\right|$ is formal, by Proposition 3.6 its $\mathbb{Q H B K S S}$ collapses at the $E^{2}$ page. Since $\mathrm{H}^{*}\left(\left|\chi^{\bullet}\right| ; \mathbb{Q}\right) \cong \mathrm{H}^{*}\left(X^{\bullet} ; \mathbb{Q}\right)$, this page coincides with that of $X^{\bullet}$, which also collapses by hypothesis. Thus $\mathrm{H}^{*}\left(\operatorname{Tot}\left(\left|\chi^{\bullet}\right|\right) ; \mathbb{Q}\right) \cong \mathrm{H}^{*}\left(\operatorname{Tot}\left(X^{\bullet}\right) ; \mathbb{Q}\right)$ and since these two totalizations are H-spaces, we deduce that they have the same rational homotopy type, and in particular the same rational homotopy groups. 
By Cartan-Serre theorem, $\chi^{\bullet} \cong \pi_{*}\left(\Omega X^{\bullet}\right) \otimes \mathbb{Q}$ so we have by [9] that $\pi_{*}\left(\left|\chi^{\bullet}\right|\right) \otimes \mathbb{Q} \cong$ $\pi_{*}\left(X^{\bullet}\right) \otimes \mathbb{Q}$. Therefore the $E^{2}$ pages of the $\mathbb{Q} \pi$ BKSS of these two cosimplicial spaces coincide. Since the spectral sequences converge to the rational homotopy groups of the totalization and those are isomorphic by the discussion above, we have that if one of these $\mathbb{Q} \pi$ BKSS collapses at the $E^{2}$ page, the same is true for the other. But the spectral sequence for $\left|\chi^{\bullet}\right|$ collapses by Corollary 3.8 .

Now fix an integer $d \geq 3$. We start with the Kontsevich operad $\mathcal{K}(\bullet)=\{\mathcal{K}(n)\}_{n \geq 0}$ in $\mathbb{R}^{d}$ as defined by Sinha in [21]. Recall that this is an operad of topological spaces homotopy equivalent to the little $d$-disks operad. In particular $\mathcal{K}(n)$ is homotopy equivalent to the configuration space of $n$ points in $\mathbb{R}^{d}$. An important additional feature is that it is a multiplicative operad in the following sense.

Definition $4.2([10,15])$. Let $(\mathcal{C}, \otimes, \mathbf{1})$ be a monoidal category where $\mathbf{1}$ is the unit object for the monoidal operation $\otimes$. A multiplicative operad $\mathcal{O}(\bullet)=\{\mathcal{O}(n)\}_{n \geq 0}$ in $\mathcal{C}$ is a non- $\Sigma$ operad (that is, an operad without the action of the symmetric groupoid or the equivariant axioms) equipped with a morphism of non- $\Sigma$ operads

$$
\mu: \mathcal{A S S}(\bullet) \longrightarrow \mathcal{O}(\bullet)
$$

where $\mathcal{A S S}(\bullet)$ is the associative non- $\Sigma$ operad defined by $\mathcal{A S S}(n)=\mathbf{1}$ for $n \geq 0$.

To a multiplicative operad $\mathcal{O}(\bullet)$, one can associate a cosimplicial object

$$
\mathcal{O}^{\bullet}=(\mathcal{O}(0) \rightleftarrows \mathcal{O}(1) \underset{\longleftrightarrow}{\rightleftarrows}(2) \cdots),
$$

where the cofaces $d^{i}: \mathcal{O}(k) \rightarrow \mathcal{O}(k+1)\left(\right.$ resp. codegeneracies $\left.s^{j}: \mathcal{O}(k) \rightarrow \mathcal{O}(k-1)\right)$ are induced by the operadic structure and the map $\mu_{2}: \mathbf{1} \rightarrow \mathcal{O}(2)\left(\right.$ resp. $\mu_{0}: \mathbf{1} \rightarrow \mathcal{O}(0)$ ) (see [15, Definition 3.1] for details). The cosimplicial space $\mathcal{K}^{\bullet}$ in equation (1) is obtained in this way from the multiplicative operad $\mathcal{K}(\bullet)$, as explained in [21].

From now on we assume $\mathbf{k}=\mathbb{Q}$ even if many of the constructions below work for more general rings. Let us consider the monoidal categories $\left(\mathrm{DGL}_{1}, \oplus, 0\right)$ and $\left(\mathrm{CDGC}_{2}, \otimes, \mathbb{Q}\right)$. Notice that the unit object is an initial object in both of these categories $\left(0\right.$ in $\mathrm{DGL}_{1}$ and $\mathbb{Q}$ in $\mathrm{CDGC}_{2}$ ), and so every non- $\Sigma$ operad in one of these categories is automatically a multiplicative operad. Applying homology to $\mathcal{K}(\bullet)$, we obtain a multiplicative operad $\mathrm{H}_{*}\left(\mathcal{K}(\bullet) ; \mathbb{Q}\right.$ ) in $\mathrm{CDGC}_{2}$ (where each DGC has zero differential). By the construction described above, we obtain a cosimplicial $\mathrm{CDGC}_{2}$

$$
\mathrm{H}_{*}(\mathcal{K} ; \mathbb{Q})^{\bullet} .
$$

On the other hand, since $\mathcal{K}(\bullet)$ is an operad of pointed spaces, we can consider its looping $\Omega \mathcal{K}(\bullet)$ which is a multiplicative operad of $\mathrm{H}$-spaces. Taking the primitive part of its homology gives a multiplicative operad $\chi(\bullet):=\mathrm{P} \mathrm{H}_{*}(\Omega \mathcal{K}(\bullet) ; \mathbb{Q})$ in $\mathrm{DGL}_{1}$ with zero differential. From this operad we get a cosimplicial $D_{G L}$

$$
\chi^{\bullet}=\mathrm{PH}_{*}(\Omega \mathcal{K} ; \mathbb{Q})^{\bullet} .
$$

Recall the Quillen functor $\mathcal{L}: \mathrm{CDGC}_{2} \rightarrow \mathrm{DGL}_{1}$ from Section 2. The cosimplicial objects (3) and (4) are related as follows.

Theorem 4.3. There exists a quasi-isomorphism of cosimplicial 1-reduced DGLs

$$
\phi^{\bullet}: \mathcal{L}\left(\mathrm{H}_{*}(\mathcal{K} ; \mathbb{Q})^{\bullet}\right) \stackrel{\simeq}{\longrightarrow} \chi^{\bullet} .
$$


Proof. By definition of $\mathcal{L}$ we have

$$
\mathcal{L}\left(\mathrm{H}_{*}(\mathcal{K}(n) ; \mathbb{Q})\right)=\left(\mathbb{L}\left(s^{-1} \mathrm{H}_{+}(\mathcal{K}(n) ; \mathbb{Q}), \partial=\partial_{1}+\partial_{2}\right)\right.
$$

where $\mathbb{L}$ is a free graded Lie algebra, $s^{-1}$ is the desuspension, $\partial_{1}=0$ because the differential of $\mathrm{H}_{*}(\mathcal{K}(n) ; \mathbb{Q})$ is zero, and $\partial_{2}$ is induced by the reduced diagonal $\bar{\Delta}[9$, $\S 22$ (a)] of the coalgebra $\mathrm{H}_{*}(\mathcal{K}(n) ; \mathbb{Q})$. Since $\mathcal{K}(n)$ has the homotopy type of the configuration space of $n$ points in $\mathbb{R}^{d}$, there is a basis $\left\{\gamma_{i j}: 1 \leq j<i \leq n\right\}$ of $\mathrm{H}_{d-1}(\mathcal{K}(n) ; \mathbf{k})$ where $\gamma_{i j}$ is the homology class representing the fundamental class of the $(d-1)$-dimensional sphere (coming from "point $i$ turning around point $j$ ") in $\mathbb{R}^{d}$, as was shown in [7]. On the other hand, Cohen and Gitler have shown in [6, Theorem $2.3]$ that there is an explicit isomorphism of DGLs

$$
\chi(n) \cong \mathbb{L}\left(B_{i j}: 1 \leq j<i \leq n\right) / I
$$

where the $B_{i j}$ are of degree $d-2$ and $I$ is the ideal generated by the infinitesimal Yang-Baxter relations:

- $\left[B_{i j}, B_{s t}\right]$ if $\{i, j\} \cap\{s, t\}=\emptyset$;

- $\left[B_{i j}, B_{i t}+(-1)^{d} B_{t j}\right]$ for $1 \leq j<t<i \leq n$; and

- $\left[B_{t j}, B_{i j}+B_{i t}\right]$ for $1 \leq j<t<i \leq n$.

Elements $B_{i j}$ are the images of the $\gamma_{i j}$ through the following desuspension and Hurewicz isomorphisms:

$$
\mathrm{H}_{d-1}(\mathcal{K}(n)) \cong \pi_{d-1}(\mathcal{K}(n)) \cong \pi_{d-2}(\Omega \mathcal{K}(n)) \cong \mathrm{P} \mathrm{H}_{d-2}(\Omega \mathcal{K}(n)) .
$$

By abuse of notation we will identify $\chi(n)$ with the right hand side of equation (5).

Consider the unique Lie algebra morphism

$$
\phi^{n}: \mathbb{L}\left(s^{-1} \mathrm{H}_{+}(\mathcal{K}(n) ; \mathbb{Q})\right) \longrightarrow \chi(n)
$$

characterized by

$$
\begin{cases}\phi^{n}\left(s^{-1} \gamma_{i j}\right)=B_{i j} & \text { for } 1 \leq j<i \leq n \\ \phi^{n}\left(s^{-1} \xi\right)=0 & \text { for } \xi \in \mathrm{H}_{+}(\mathcal{K}(n) ; \mathbb{Q}) \text { with } \operatorname{deg}(\xi) \neq d-1\end{cases}
$$

We check that $\phi^{n}$ commutes with the differentials. Recall that $\mathrm{H}_{+}(\mathcal{K}(n) ; \mathbb{Q})$ is concentrated in degrees $d-1,2(d-1), 3(d-1)$, etc. Since for each element $\eta \in$ $\mathrm{H}_{+}(\mathcal{K}(n) ; \mathbb{Q})$ of degree $>2(d-1)$ each term of the reduced diagonal of $\eta$ contains a factor of degree $>d-1$ and since $\phi^{n}\left(s^{-1} \xi\right)=0$ for $\operatorname{deg}(\xi)>d-1$, it is enough to show that $\phi^{n}\left(\partial_{2}\left(s^{-1} \xi\right)\right)=0$ when $\operatorname{deg}(\xi)=2(d-1)$.

The fact that $\phi^{n}\left(\partial_{2}\left(s^{-1} \xi\right)\right)=0$ when $\operatorname{deg}(\xi)=2(d-1)$ is a computation using the explicit form of the reduced diagonal (which is related to the Arnold or 3-term relation) and the infinitesimal Yang-Baxter relations. We now illustrate the computation in a special case.

The cohomology algebra $\mathrm{H}^{*}(\mathcal{K}(n) ; \mathbb{Q})$ is generated by elements $A_{i j}$ dual to $\gamma_{i j}$, for $1 \leq j<i \leq n$, subject to the relations $A_{i j}^{2}=0$ and $A_{i j} A_{i k}=A_{k j} A_{i k}-A_{k j} A_{i j}$ for $1 \leq j<k<i \leq n$ [5, page 22]. The latter relation is called the three-term relation. We specialize to the case $n=3$. A basis of $\mathrm{H}^{2(d-1)}(\mathcal{K}(3), \mathbb{Q})$ is $\left\{A_{21} A_{31}, A_{21} A_{32}\right\}$, and the three-term relation is $A_{31} A_{32}=A_{21} A_{32}-A_{21} A_{31}$. We denote the dual basis of $\mathrm{H}_{2(d-1)}(\mathcal{K}(3), \mathbb{Q})$ by $\left\{\xi_{21,31}, \xi_{21,32}\right\}$. The diagonal $\Delta \xi$ is computed using the adjunction $\langle\Delta \xi, \alpha \otimes \beta\rangle=\langle\xi, \alpha . \beta\rangle$ for cohomology classes $\alpha$ and $\beta$. Notice that $\langle\Delta \xi, \beta \otimes \alpha\rangle=(-1)^{d-1}\langle\Delta \xi, \alpha \otimes \beta\rangle$ when $\alpha, \beta \in\left\{A_{i j}\right\}$. Evaluating $\Delta \xi_{21,31}$ on the basis 
$\left\{A_{i j} \otimes A_{u v}: 1 \leq j<i \leq 3,1 \leq v<u \leq 3\right\}$, which is dual to $\left\{(-1)^{d-1} \gamma_{i j} \otimes \gamma_{u v}\right\}$, we get

$$
\Delta \xi_{21,31}=\left(\gamma_{21} \otimes \gamma_{31}+(-1)^{d-1} \gamma_{31} \otimes \gamma_{21}\right)-\left(\gamma_{31} \otimes \gamma_{32}+(-1)^{d-1} \gamma_{32} \otimes \gamma_{31}\right) .
$$

Using $[9, \S 22(\mathrm{e})]$ we compute

$$
\partial_{2}\left(s^{-1} \xi_{21,31}\right)=\left[s^{-1} \gamma_{21}, s^{-1} \gamma_{31}\right]-\left[s^{-1} \gamma_{31}, s^{-1} \gamma_{32}\right],
$$

so $\phi^{n}\left(\partial_{2}\left(s^{-1}\left(\xi_{21,31}\right)\right)=\left[B_{21}, B_{31}\right]-\left[B_{31}, B_{32}\right]\right.$ which is zero by the last two YangBaxter relations. A similar computation shows that

$$
\phi^{n}\left(\partial_{2}\left(s^{-1} \xi_{21,32}\right)\right)=0 .
$$

By symmetry we deduce from these two examples that for all $n \geq 3$, we have $\phi^{n}\left(\partial_{2}\left(s^{-1} \xi_{p q, r s}\right)\right)=0$ when $\{p, q\} \cap\{r, s\}$ is a singleton. When $\{p, q\} \cap\{r, s\}$ is empty we also obtain the desired relation using the first Yang-Baxter relation.

Since $s^{-1} \gamma_{i j}$ are cycles that surject to the generators $B_{i j}$ of $\chi(n)$ it is clear that $\phi^{n}$ induces a surjection in homology. On the other hand by Kontsevich's theorem [12, Theorem 2] (see also [14]) the spaces $\mathcal{K}(n)$ are formal over $\mathbb{R}$. This implies that

$$
\pi_{*}(\Omega \mathcal{K}(n)) \otimes \mathbb{R} \cong \mathrm{H}_{*}\left(\mathcal{L}\left(\mathrm{H}_{*}(\mathcal{K}(n) ; \mathbb{R})\right)\right)
$$

as graded vector spaces. By Cartan-Serre Theorem the left side of equation (7) is isomorphic to $\mathrm{PH}_{*}(\Omega \mathcal{K}(n) ; \mathbb{R})$. This result is still true if we replace $\mathbb{R}$ by $\mathbb{Q}$. Thus we deduce that the graded vector spaces $\chi(n)$ and $\mathrm{H}_{*}\left(\mathcal{L}\left(\mathrm{H}_{*}(\mathcal{K}(n) ; \mathbb{Q})\right)\right.$ are isomorphic. Since they are of finite type and $\mathrm{H}\left(\phi^{n}\right)$ is surjective, we get that $\phi^{n}$ is a quasiisomorphism.

It remains to prove that $\phi^{\bullet}=\left\{\phi^{n}\right\}_{n \geq 0}$ commutes with the cofaces and codegeneracies. Since all the maps are maps of Lie algebras it is enough to check commutativity on the generators $s^{-1} \xi$ for $\xi \in \mathrm{H}_{+}(\mathcal{K}(n) ; \mathbf{k})$. If $\operatorname{deg}(\xi)>d-1$, this is immediate since every element of degree $>d-1$ is sent to 0 by $\phi^{n}$. For elements of degree $d-1$, notice that in that degree $\phi^{n}$ can be identified with the desuspension of the Hurewicz map as in isomorphism (6), and since the Hurewicz map is natural, it commutes with the maps induced by the cofaces and codegeneracies.

Corollary 4.4. The pointed cosimplicial space $\left|\chi^{\bullet}\right|$ is formal and coformal over $\mathbb{Q}$.

Proof. Coformality is clear from the first part of Lemma 2.1 since $\chi^{\bullet}$ is by definition a cosimplicial 1-reduced DGL with zero internal differentials. Also recall from Lemma 2.1 that the realization functor $|-|: \mathrm{DGL}_{1} \rightarrow$ Top preserves weak equivalences. Therefore by Theorem 4.3 it is enough to show that

$$
\left|\mathcal{L}\left(\mathrm{H}_{*}(\mathcal{K}(-) ; \mathbb{Q})^{\bullet}\right)\right|:=\left|\# \mathcal{C} \mathcal{L}\left(\mathrm{H}_{*}(\mathcal{K}(-) ; \mathbb{Q})^{\bullet}\right)\right|
$$

is formal. By Lemma 2.1, if $C$ is a 2-reduced CDGC, we have a chain of natural quasiisomorphisms $A_{P L}(|\# \mathcal{C} \mathcal{L}(C)|) \simeq \# \mathcal{C} \mathcal{L}(C)$ and $\# \mathcal{C} \mathcal{L}(C)$ is naturally weakly equivalent to $\# C$ by $\left[9\right.$, Theorem 22.9]. All of this and Theorem 4.3 imply that $A_{P L}\left(\left|\chi^{\bullet}\right|\right)$ is naturally weakly equivalent to $\# \mathrm{H}_{*}(\mathcal{K}(-) ; \mathbb{Q})^{\bullet}$.

We can now prove the main result of the paper. 
Proof of Theorem 1.1. We show that the hypotheses of Theorem 4.1 hold for $X^{\bullet}=$ $\mathcal{K}^{\bullet}$. Indeed by Theorem 4.3 and Corollary 4.4 , the cosimplicial space $\left|\chi^{\bullet}\right|$ is formal and has the same rational cohomology as $\mathcal{K}^{\bullet}$. The main result of [13] establishes that the $\mathbb{Q}$ HBKSS of $\mathcal{K}^{\bullet}$ collapses at the $E^{2}$ page for $d \geq 4$. The spectral sequences converge by arguments analogous to [21, Theorem 2.1 or 7.2$]$ and their abutments are of finite type. Finally the totalizations of $\mathcal{K}^{\bullet}$ and $\left|\chi^{\bullet}\right|$ are algebras over the little 2 -disks operad by [15, Theorem 3.3] and, since these totalizations are connected, they are H-spaces. So by Theorem 4.1, the $\mathbb{Q} \pi$ BKSS associated to $\mathcal{K}^{\bullet}$ collapses at the $E^{2}$ page.

\section{Acknowledgements}

We thank Yves Félix for help with computations.

\section{References}

[1] G. Arone, P. Lambrechts, and I. Volić, Calculus of functors, operad formality and rational homology of embedding spaces, to appear in Acta Mathematica. arXiv:math/0607486.

[2] A. K. Bousfield, On the homology spectral sequence of a cosimplicial space, Amer. J. Math. 109 (1987), no. 2, 361-394.

[3] A. K. Bousfield and V. K. A. M. Gugenheim, On PL de Rham theory and rational homotopy type, Mem. Amer. Math. Soc. 8 (1976), no. 179, ix+94.

[4] A. K. Bousfield and D. M. Kan, Homotopy limits, completions and localizations, SpringerVerlag, Berlin (1972). Lecture Notes in Mathematics, Vol. 304.

[5] F. R. Cohen, On configuration spaces, their homology, and Lie algebras, J. Pure Appl. Algebra 100 (1995), no. 1-3, 19-42.

[6] F. R. Cohen and S. Gitler, On loop spaces of configuration spaces, Trans. Amer. Math. Soc. 354 (2002), no. 5, 1705-1748 (electronic).

[7] F. R. Cohen, T. J. Lada, and J. P. May, The homology of iterated loop spaces, Springer-Verlag, Berlin (1976). Lecture Notes in Mathematics, Vol. 533.

[8] P. Deligne, P. Griffiths, J. Morgan, and D. Sullivan, Real homotopy theory of Kähler manifolds, Invent. Math. 29 (1975), no. 3, 245-274.

[9] Y. Félix, S. Halperin, and J.-C. Thomas, Rational homotopy theory, Vol. 205 of Graduate Texts in Mathematics, Springer-Verlag, New York (2001), ISBN 0-387-95068-0.

[10] M. Gerstenhaber and A. A. Voronov, Homotopy G-algebras and moduli space operad, Internat. Math. Res. Notices (1995), no. 3, 141-153 (electronic).

[11] T. G. Goodwillie, J. R. Klein, and M. S. Weiss, Spaces of smooth embeddings, disjunction and surgery, in Surveys on surgery theory, Vol. 2, Vol. 149 of Ann. of Math. Stud., 221-284, Princeton Univ. Press, Princeton, NJ (2001).

[12] M. Kontsevich, Operads and motives in deformation quantization, Lett. Math. Phys. 48 (1999), no. 1, 35-72. Moshé Flato (1937-1998).

[13] P. Lambrechts, V. Turchin, and I. Volić, The rational homology of spaces of long knots in codimension $>2$. Submitted. arXiv:math.AT/0703649.

[14] P. Lambrechts and I. Volić, Formality of the little d-discs operad. In preparation. Available at http://palmer.wellesley.edu/ ivolic/pages/papers.html.

[15] J. E. McClure and J. H. Smith, A solution of Deligne's Hochschild cohomology conjecture, in Recent progress in homotopy theory (Baltimore, MD, 2000), Vol. 293 of Contemp. Math., 153-193, Amer. Math. Soc., Providence, RI (2002).

[16] J. Neisendorfer and T. Miller, Formal and coformal spaces, Illinois J. Math. 22 (1978), no. 4, $565-580$.

[17] D. G. Quillen, Rational homotopy theory, Ann. of Math. (2) 90 (1969) 205-295.

[18] K. P. Scannell and D. P. Sinha, A one-dimensional embedding complex, J. Pure Appl. Algebra 170 (2002), no. 1, 93-107. 
[19] S. Schwede and B. Shipley, Stable model categories are categories of modules, Topology 42 (2003), no. 1, 103-153.

[20] D. Sinha, The topology of spaces of knots. Submitted. math.AT/0202287, version 6 (2007).

[21] D. P. Sinha, Operads and knot spaces, J. Amer. Math. Soc. 19 (2006), no. 2, 461-486 (electronic).

Department of Mathematics, University of Virginia, P. O. Box 400137 Charlottesville, VA $22904-4137$

E-mail address: zga2m@virginia.edu

$U R L:$ http://www.math.virginia.edu/ zga2m/

Institut Mathématique, Université Catholique de Louvain, 2 Chemin du Cyclotron, B1348 Louvain-la-Neuve, Belgium

E-mail address: pascal.lambrechts@uclouvain.be

$U R L:$ http://milnor.math.ucl.ac.be/plwiki

Université Catholique de louvain, Belgium, and Department of Mathematics, Kansas State University, Manhattan, KS 66506, USA.

E-mail address: turchin@math.ksu.edu

$U R L:$ http://www.math.ksu.edu/ ${ }^{\text {turchin }}$

Department of Mathematics, Wellesley College, 106 Central Street, Wellesley, Ma 02481

E-mail address: ivolic@wellesley.edu

$U R L:$ http://palmer.wellesley.edu/ ${ }^{\sim}$ ivolic 